\title{
Biostimulant Nanoencapsulation: The New Keystone To Fight Hunger
}

\author{
David Jiménez-Arias, * Sarai Morales-Sierra, Andrés A. Borges, and David Díaz Díaz*
}

Cite This: J. Agric. Food Chem. 2020, 68, 7083-7085

Read Online

\section{ACCESS | Llll Metrics \& More}

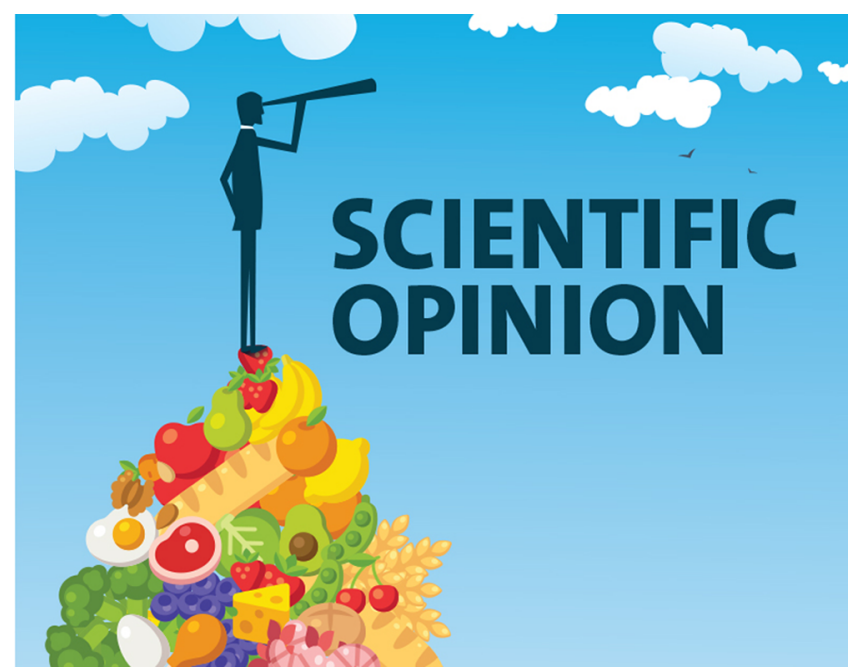

T $\mathrm{n}$ a challenging climate change scenario, we need to provide 1 agriculture with new tools to improve food crop yields, to feed a growing population that is expected to reach 9 billion in 2050. Thus, agricultural production will need to double to ensure global food security in the near future. Unfortunately, production is nowadays not increasing but rather decreasing in many countries and regions around the world. In this regard, plant stresses are the primary cause of crop yield losses, which, in many cases, are higher than net production. During the 21 st century, these negative effects are predicted to rise as a result of global warming. Importantly, agriculture and climate change are closely correlated in various ways. Indeed, climate change is currently the main cause of biotic and abiotic stresses, which have local adverse effects on agriculture. This leads to a tough future scenario but constitutes a unique opportunity for multidisciplinary research to solve this problem by developing new green and sustainable approaches.

A hot topic in this field is the use of biostimulants (Bs), defined as substances that are used to enhance plant growth other than fertilizers or pesticides. Bs are commonly ecofriendly substances and inert for the ecosystems, and their utilization is widely reported in the literature as enhancing tolerance against stress or increasing crop production. A good example is the utilization of amino acids ${ }^{1}$ that lower water consumption in the field. However, weekly treatments are required to be effective, which becomes prohibitively expensive on large farms. One of the main reasons is probably the easy degradability of Bs by soil microorganisms, which shortens their activity period. A promising strategy to solve this issue involves the use of nanoencapsulation techniques. In this approach, active substances are enclosed within an inert nanomaterial that protects them against environmental degradation and/or provides a controlled release. This process is already in use mainly with pesticides and fertilizers, but it is not yet widely explored with Bs, for which only a few recent trials have been reported in the last 2 years. It is worth emphasizing that Bs should be non-toxic, which constitutes an important quality that must always be tested in any new potential Bs for its correct assessment.

One of the most desired qualities of a commercial formulation is to increase the half-life of the active substance in the soil. Within this context, nanoencapsulation offers two different strategies based on either the possibilities of continuous controlled release over time or protecting the active material inside the particle to prevent natural degradation. Kumaraswamy and co-workers ${ }^{2}$ have described the use of salicylic acid (SA)-functionalized chitosan nanoparticles (NPs) prepared by an ionic gelation method. In this report, the concentrations of chitosan and sodium tripolyphosphate were adjusted to 0.4 and $0.2 \%(\mathrm{w} / \mathrm{v})$ to deliver SA $(0.1 \%, \mathrm{w} / \mathrm{v})$ as a Bs. These NPs were found to promote plant defenses against Fusarium verticillioides in maize, controlling the release of $80.4 \%$ SA during a 7 day period. Moreover, that encapsulation formula provided better enhanced growth and antioxidant response under disease conditions compared to the treatment of SA alone. Meanwhile, Sharma et al.3 used the same synthesis method but a different composition of chitosan $(0.5 \%, \mathrm{w} / \mathrm{v})$ and tripolyphospate $(0.25 \%, \mathrm{w} / \mathrm{v})$ to entrap and stabilize the same SA concentration $(0.1 \%, \mathrm{w} / \mathrm{v})$. This formulation released nearly $30 \%$ SA in the first $24 \mathrm{~h}$. However, after this point, only 5\% SA was liberated during a 10 day study period. The authors claimed that these NPs are easily degraded by soil microorganism enzymes, providing an excellent carrier

Received: May 8, 2020

Published: June 26, 2020 


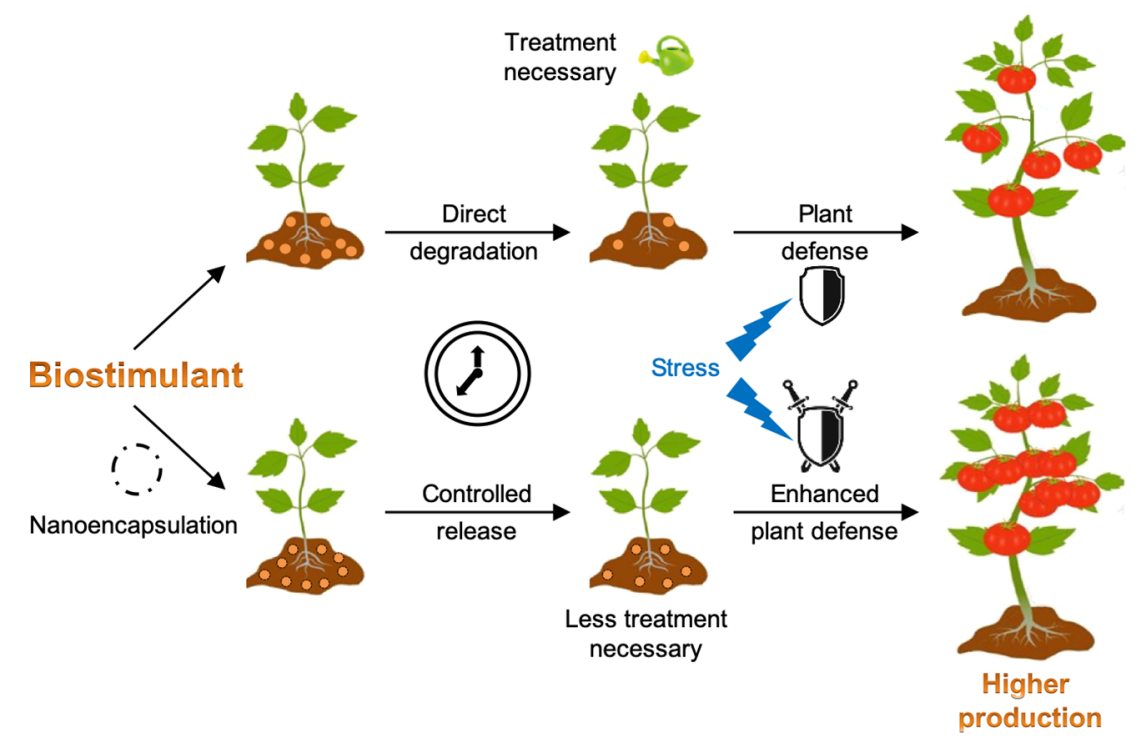

Figure 1. Illustration of the main benefits of biostimulant nanoencapsulation on plant production.

to enhance growth and antioxidant response in Zea mays plants.

Another interesting application is the nanoencapsulation of active compounds, which are otherwise impossible to use alone as a result of their inherent environmental instability. For instance, nitric oxide is a signaling molecule involved in plant response against various environmental stresses, but its use with donors is hampered by their rapid degradation in the field. In this regard, Oliveira and co-workers ${ }^{4}$ have reported the use of mercaptosuccinic acid, a NO donor precursor with high chemical instability and great prospective as Bs. Encapsulation with chitosan $\left(2.6 \mathrm{mg} \mathrm{mL}^{-1}\right)$ and sodium tripolyphosphate $\left(0.6 \mathrm{mg} \mathrm{mL}^{-1}\right)$ NPs through an ionotropic gelation process was found to prevent the degradation issue and facilitate the plant treatment to increase salt tolerance, ${ }^{4}$ thus reducing the deleterious effect of $\mathrm{NaCl}$ in rice.

Finally, the major reward from the use of Bs will be the production growth to build a more profitable farm business based on an optimal cost/productivity ratio. Let us consider the case of giberellic acid, a plant hormone that is able to increase fruit production by $101 \%$ in tomato plants. Pereira et al. $^{5}$ assessed NPs made of chitosan $(0.07 \%, \mathrm{w} / \mathrm{v})$ and sodium alginate $(0.063 \%, \mathrm{w} / \mathrm{v})$ with enclosed giberellic acid $(0.05 \mathrm{mg}$ $\left.\mathrm{mL}^{-1}\right)$. These NPs were found to increase the production of tomato fruits by $77 \%$, also enhancing the growth parameters. It is noteworthy that its manufacture process does not involve the use of organic solvents, being particularly advantageous in sustainable agriculture.

In our opinion, biostimulant substances constitute one of the next generation of tools for improving agricultural practices. In fact, it is estimated that the market based on these substances will increase by $12 \%$ per year to over $\$ 2200$ million by 2018 . They constitute a demonstrated way to increase yield and tolerance against biotic and abiotic stresses in an eco-friendly way. However, one of the fundamental pillars of Bs is their easy degradation in the field, which prevents bioaccumulation. Unfortunately, this means they have to be continuously administered, hindering profitability in the field.

Herein, we have described some of the benefits of entrapping $\mathrm{Bs}$ in nanocarriers to control their release or protect them from degradation, thus increasing crop yield
(Figure 1). Despite the benefits of the nanoencapsulation of Bs that we have highlighted in this "viewpoint", more extensive research is still necessary to spread this approach worldwide. This will help make the utilization of Bs cost-effective, providing farmers with improved and efficient tools to combat future food shortages as a result of global climate change.

\section{AUTHOR INFORMATION}

\section{Corresponding Authors}

David Jiménez-Arias - Department of Agrobiology, Instituto de Productos Naturales y Agrobiologia (IPNA)-Consejo Superior de Investigaciones Cientificas (CSIC), 38306 San Cristóbal de

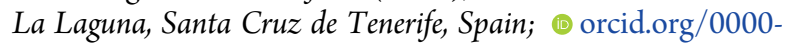
0003-2304-7373; Email: david.j.a1983@gmail.com

David Díaz Díaz - Departamento de Quimica Orgánica and Instituto Universitario de Bio-Orgánica Antonio González, Universidad de La Laguna, 38306 San Cristóbal de La Laguna, Santa Cruz de Tenerife, Spain; Institut für Organische Chemie, Universität Regensburg, 93040 Regensburg, Germany; ○ orcid.org/0000-0002-0557-3364; Email: ddiazdiaz@ ull.edu.es

\section{Authors}

Sarai Morales-Sierra - Department of Agrobiology, Instituto de Productos Naturales y Agrobiologia (IPNA)-Consejo Superior de Investigaciones Cientificas (CSIC), 38306 San Cristóbal de La Laguna, Santa Cruz de Tenerife, Spain

Andrés A. Borges - Department of Agrobiology, Instituto de Productos Naturales y Agrobiologia (IPNA)-Consejo Superior de Investigaciones Cientificas (CSIC), 38306 San Cristóbal de La Laguna, Santa Cruz de Tenerife, Spain

Complete contact information is available at:

https://pubs.acs.org/10.1021/acs.jafc.0c02872

\section{Funding}

This research was financially supported by Programa de Cooperación INTEREG MAC 2014-2020: MAC2/1.1b/279. David Díaz Díaz thanks the Spanish Ministry of Science, Innovation and Universities for the Senior Beatriz Galindo Award (BEAGAL18/00166, Distinguished Researcher at the University of La Laguna). 


\section{Notes}

The authors declare no competing financial interest.

\section{ACKNOWLEDGMENTS}

D.D.D. thanks NANOtec, INTech, Cabildo de Tenerife and ULL for laboratory facilities. Language editing has been carried out by Guido Jones, currently funded by the Cabildo de Tenerife, under the TFinnova Programme supported by MEDI and FDCAN funds.

\section{REFERENCES}

(1) Jiménez-Arias, D.; García-Machado, J. A.; Morales-Sierra, S.; Luis, J. C.; Suarez, E.; Hernández, M.; Valdés, F.; Borges, A. A. Lettuce plants treated with L-pyroglutamic acid increase yield under water deficit stress. Environ. Exp. Bot. 2019, 158, 215-222.

(2) Kumaraswamy, R. V.; Kumari, S.; Choudhary, R. C.; Sharma, S. S.; Pal, A.; Raliya, R.; Biswas, P.; Saharan, V. Salicylic acid functionalized chitosan nanoparticle: A sustainable biostimulant for plant. Int. J. Biol. Macromol. 2019, 123, 59-69.

(3) Sharma, G.; Kumar, A.; Devi, K. A.; Prajapati, D.; Bhagat, D.; Pal, A.; Raliya, R.; Biswas, P.; Saharan, V. Chitosan nanofertilizer to foster source activity in maize. Int. J. Biol. Macromol. 2020, 145, 226234.

(4) Oliveira, H. C.; Gomes, B. C. R.; Pelegrino, M. T.; Seabra, A. B. Nitric oxide-releasing chitosan nanoparticles alleviate the effects of salt stress in maize plants. Nitric Oxide 2016, 61, 10-19.

(5) Pereira, A. E. S.; Oliveira, H. C.; Fraceto, L. F. Polymeric nanoparticles as an alternative for application of gibberellic acid in sustainable agriculture: A field study. Sci. Rep. 2019, 9, 7135. 